\title{
Antiangiogenic Therapy for Patients with Recurrent and Newly Diagnosed Malignant Gliomas
}

\author{
Katsuyuki Shirai, Michael R. Siedow, and Arnab Chakravarti \\ Department of Radiation Oncology, The Ohio State University Comprehensive Cancer Center, Columbus, OH 43210, USA \\ Correspondence should be addressed to Arnab Chakravarti, arnab.chakravarti@osumc.edu
}

Received 1 May 2011; Accepted 24 May 2011

Academic Editor: Arkadiusz Dudek

Copyright ( $) 2012$ Katsuyuki Shirai et al. This is an open access article distributed under the Creative Commons Attribution License, which permits unrestricted use, distribution, and reproduction in any medium, provided the original work is properly cited.

\begin{abstract}
Malignant gliomas have a poor prognosis despite advances in diagnosis and therapy. Although postoperative temozolomide and radiotherapy improve overall survival in glioblastoma patients, most patients experience a recurrence. The prognosis of recurrent malignant gliomas is dismal, and more effective therapeutic strategies are clearly needed. Antiangiogenesis is currently considered an attractive targeting therapy for malignant gliomas due to its important role in tumor growth. Clinical trials using bevacizumab have been performed for recurrent glioblastoma, and these studies have shown promising response rates along with progressionfree survival. Based on the encouraging results, bevacizumab was approved by the FDA for the treatment of recurrent glioblastoma. In addition, bevacizumab has shown to be effective for recurrent anaplastic gliomas. Large phase III studies are currently ongoing to demonstrate the efficacy and safety of the addition of bevacizumab to temozolomide and radiotherapy for newly diagnosed glioblastoma. In contrast, several other antiangiogenic drugs have also been used in clinical trials. However, previous studies have not shown whether antiangiogenesis improves the overall survival of malignant gliomas. Specific severe side effects, difficult assessment of response, and lack of rational predictive markers are challenging problems. Further studies are warranted to establish the optimized antiangiogenesis therapy for malignant gliomas.
\end{abstract}

\section{Introduction}

Malignant gliomas such as glioblastoma and anaplastic gliomas are the most common primary brain tumors in adults [1]. Temozolomide and radiotherapy have been demonstrated to improve overall survival in glioblastoma patients [2-4]. Despite advances in diagnosis and therapy, prognosis remains poor with a median overall survival of 12 to 15 months in glioblastoma due to the resistance to radiotherapy and chemotherapy. Although anaplastic gliomas tend to respond well to these treatments, the median survival time is 2 to 3 years $[5,6]$. The prognosis of recurrent malignant gliomas is dismal with the median overall survival and progression-free survival (PFS) of 7.5 months and 2.5 months, respectively [7]. More effective therapeutic strategies are needed for these patients.

Malignant gliomas are characterized by vascular proliferation or angiogenesis $[8,9]$. Vascular endothelial growth factor (VEGF) is highly expressed in glioblastoma and has been shown to regulate tumor angiogenesis [10]. Bevacizumab was developed as a humanized monoclonal antibody against VEGF. Clinical trials of recurrent glioblastoma showed benefits of bevacizumab in response rate and PFS [11-13]. Based on these favorable results, bevacizumab was approved by the US Food and Drug Administration (FDA) for recurrent glioblastoma. For newly diagnosed glioblastoma, phase II trials showed that the addition of bevacizumab to temozolomide and radiotherapy improves PFS [14, 15]. Other antiangiogenic drugs have also been investigated and used in several clinical studies [16]. In this paper, we focus on biological and clinical findings of antiangiogenesis therapy for malignant gliomas.

\section{Biological Aspects of Antiangiogenic Therapy for Glioblastoma}

Advances in molecular biology have provided pathogenesis of malignant gliomas. Several clinical and preclinical studies 
proposed that tumor-related blood vessel, called "angiogenesis", is required for solid tumor growth, including malignant gliomas $[10,16]$. Endothelial proliferation is a marker of histological grading systems for malignant gliomas because of an association between a degree of microvascularity and biologic aggressiveness [17]. Glioblastoma is particularly characterized by vascular proliferation and the extent of necrosis. These findings indicate that tumor antiangiogenesis is a promising candidate to inhibit the growth of malignant gliomas. VEGF, a critical mediator of angiogenesis, has emerged as a novel target of antiangiogenic therapy. Glioblastoma cells have been shown to secrete VEGF, resulting in the endothelial proliferation and tumor survival in vivo [18]. VEGF is expressed in malignant gliomas and is associated with tumor grade and vascularity $[19,20]$. Therefore, it is postulated that antiangiogenesis suppresses blood flow and inhibits the tumor growth. Monoclonal antibodies against VEGF were shown to inhibit the growth of glioma cells in vivo [21]. A VEGF inhibitor directly affects glioma stem cells that are more resistant to chemotherapy and radiotherapy [22]. Furthermore, antiangiogenesis can normalize tumor vasculature and decrease interstitial fluid pressure, providing an improved delivery of chemotherapeutics and oxygen. Consequently, antiangiogenesis is expected to work synergistically with radiotherapy and chemotherapy $[23,24]$. Given these findings, VEGF inhibitors are expected to be a novel antiangiogenic therapy for malignant gliomas.

\section{The Efficacy and Safety of Bevacizumab for Recurrent Malignant Gliomas}

3.1. Bevacizumab for Recurrent Glioblastoma. Bevacizumab was developed as a humanized monoclonal antibody to bind VEGF-A, preventing the interaction and activation of VEGF receptor tyrosine kinases $[25,26]$. This drug is approved by the FDA and is in clinical use for the treatment of colorectal cancer, nonsmall cell lung cancer, breast cancer, renal cell carcinoma, and glioblastoma [27]. Table 1 shows several clinical studies of bevacizumab for recurrent malignant glioma patients.

The first phase II trial for 35 recurrent glioblastoma was performed to investigate the efficacy of intravenous administration of bevacizumab and irinotecan, a topoisomerase 1 inhibitor [11]. The 6-month PFS was 46\%, and median overall survival was 10.5 months, respectively. At least a partial response was observed in $57 \%$ of patients. A larger, randomized, noncomparative phase II study, called the BRAIN study, was performed using bevacizumab with or without irinotecan for 167 recurrent glioblastoma. In this study, the response rates were $28.2 \%$ and $37.8 \%$, and 6-month PFS were $42.6 \%$ and $50.3 \%$ in bevacizumab alone and bevacizumab plus irinotecan groups, respectively [12]. Another phase II study of bevacizumab alone for 48 recurrent glioblastomas showed that response rate was 35\% and 6-month PFS was 29\%, respectively [13]. These results were more favorable than a previous database of 8 negative trials having 6-month PFS of $15 \%$ for recurrent glioblastoma patients [7]. Furthermore, an additional advantage of bevacizumab is its ability to decrease tumor edema and to reduce steroid dose. Given the efficacy of bevacizumab for recurrent glioblastoma in the clinical setting, bevacizumab monotherapy has since been approved by the FDA.

3.2. Toxicity Profile of Bevacizumab. Since VEGF plays an important role in vascular function and physiological angiogenesis, its inhibition via bevacizumab has been reported to cause serious adverse events [34]. The first phase II study of recurrent glioblastoma treated by bevacizumab and irinotecan reported that five patients (14\%) discontinued treatment due to central nervous system (CNS) hemorrhage, deep venous thrombosis, and pulmonary emboli [11]. No fatal adverse events were reported in this study. BRAIN study reported the detailed information on adverse events of bevacizumab [12]. Grade $\geq 3$ adverse events were observed in $65.8 \%$ and $46.4 \%$ of bevacizumab plus irinotecan and bevacizumab alone groups, respectively. The most common causes of grade $\geq 3$ adverse events were convulsion, hypertension, neutropenia, and fatigue. CNS hemorrhages of any grades were observed in five patients (3.0\%). There were one fatal adverse event $(1.3 \%)$ in bevacizumab plus irinotecan group and two $(2.3 \%)$ in bevacizumab alone group, respectively. Selecting appropriate patients, early assessment of toxicity, and adequate management should be required to reduce the serious adverse events of bevacizumab.

Arterial and venous thromboses are generally reported in treatment of antiangiogenesis therapy, although the inherent risk of these thromboses is higher among malignant glioma patients. A retrospective study of 9849 patients with malignant gliomas showed that 2-year cumulative incidence of symptomatic venous thromboembolism was 7.5\% [35]. Further studies are necessary to evaluate the additional risk of thrombosis in malignant glioma patients treated by bevacizumab.

Recently, a meta-analysis of randomized control trials in several tumor types showed that bevacizumab in combination with chemotherapy increases fatal adverse events when compared with chemotherapy alone [36]. The overall incidence of fatal adverse events was $2.5 \%$ in bevacizumab therapy with the common causes being hemorrhage, neutropenia, and gastrointestinal tract perforation. Interestingly, the type of chemotherapeutic agents was significantly associated with relative risk of fatal adverse events. The addition of bevacizumab was associated with increased fatal adverse events in patients receiving taxanes or platinum agents $(3.3 \%$ versus $1.0 \%$ ) but not in those receiving other agents $(0.8 \%$ versus $0.9 \%$ ). In clinical trials of malignant gliomas, the addition of irinotecan or temozolomide to bevacizumab has often been performed and may be associated with lower fatal adverse events. However, this meta-analysis did not include the trials of brain tumors, and further investigations are required to evaluate the fatal adverse events of bevacizumab and chemotherapy in malignant gliomas.

3.3. Bevacizumab for Recurrent Anaplastic Gliomas. Anaplastic gliomas have a slightly better prognosis than glioblastoma [37], and the median survival time is 2 to 3 years. However, there are no standard treatments for progression or recurrence of anaplastic gliomas, and a novel treatment 
TABLE 1: Bevacizumab for recurrent or newly malignant gliomas.

\begin{tabular}{|c|c|c|c|c|c|c|c|}
\hline Study & Agents & Patients & $\mathrm{RR}$ & MPFS & 6-PFS & MST & Ref. \\
\hline Phase II & Bevacizumab + irinotecan & 35 recurrent GBM & $57 \%$ & 6 months & $46 \%$ & 10.5 months & {$[11]$} \\
\hline \multirow{2}{*}{ Phase II } & Bevacizumab & 85 recurrent GBM & $28 \%$ & 4.2 months & $43 \%$ & 9.2 months & \multirow{2}{*}[12]{} \\
\hline & Bevacizumab + irinotecan & 82 recurrent GBM & $38 \%$ & 5.6 months & $50 \%$ & 8.7 months & \\
\hline Phase II & Bevacizumab & 48 recurrent GBM & $35 \%$ & 4 months & $29 \%$ & 7.7 months & [13] \\
\hline \multirow{2}{*}{ Phase II } & \multirow{2}{*}{ Bevacizumab + irinotecan } & 23 recurrent GBM & $61 \%$ & 5.0 months & $30 \%$ & 10 months & \multirow{2}{*}{28} \\
\hline & & 9 recurrent AG & $67 \%$ & 7.5 months & $56 \%$ & Not reached & \\
\hline Phase II & Bevacizumab + irinotecan & 33 recurrent $A G$ & $61 \%$ & 7.5 months & $55 \%$ & 16.3 months & [29] \\
\hline \multirow{2}{*}{ Retrospective } & \multirow{2}{*}{ Bevacizumab + SRT } & 20 recurrent GBM & $50 \%$ & 7.3 months & $65 \%$ & 12.5 months & \multirow[t]{2}{*}[30]{} \\
\hline & & 5 recurrent $A G$ & $60 \%$ & 7.5 months & $60 \%$ & 16.5 months & \\
\hline \multirow{2}{*}{ Retrospective } & SRS + bevacizumab & \multirow{2}{*}{49 recurrent GBM } & - & 5.2 months & - & 11.2 months & \multirow{2}{*}[31]{} \\
\hline & SRS + other drugs & & - & 2.1 months & - & 3.9 months & \\
\hline \multirow{2}{*}{ Phase II } & \multirow[t]{2}{*}{ Bevacizumab + erlotinib } & 25 recurrent GBM & $48 \%$ & 4.5 months & $28 \%$ & 10.5 months & \multirow{2}{*}[32]{} \\
\hline & & 32 recurrent $A G$ & $31 \%$ & 5.9 months & $44 \%$ & 17.8 months & \\
\hline Phase II & Bevacizumab + RT/TMZ & $\begin{array}{c}70 \text { newly diagnosed } \\
\text { GBM }\end{array}$ & - & 13.6 months & $88 \%$ & 19.6 months & {$[14]$} \\
\hline Phase II & Bevacizumab + RT/TMZ & $\begin{array}{c}125 \text { newly diagnosed } \\
\text { GBM }\end{array}$ & - & 13.8 months & $87 \%$ & - & {$[15]$} \\
\hline Phase II & $\begin{array}{l}\text { Adjuvant bevacizumab } \\
+ \text { irinotecan }+\mathrm{TMZ}\end{array}$ & $\begin{array}{c}125 \text { newly diagnosed } \\
\text { GBM }\end{array}$ & - & 13.8 months & - & 21.3 months & {$[33]$} \\
\hline
\end{tabular}

RR: response rate; MPFS: median progression-free survival; 6-PFS: 6-month progression-free survival; MST: median overall survival time; GBM: glioblastoma multiforme; AG: anaplastic gliomas; SRT: stereotactic radiotherapy; SRS: stereotactic radiosurgery; RT: radiotherapy; TMZ: temozolomide.

strategy is needed. Recent studies have shed light on the antiangiogenic therapy in recurrent anaplastic gliomas. A phase II study of bevacizumab and irinotecan was performed in 23 glioblastoma and 9 anaplastic gliomas [28]. The response rates were $61 \%$ and $67 \%$, and 6 -month PFS were $30 \%$ and $56 \%$ in glioblastoma and anaplastic gliomas, respectively. Another phase II trial was conducted for 33 anaplastic gliomas treated by bevacizumab and irinotecan [29]. This study included 25 anaplastic astrocytomas and 8 anaplastic oligodendrogliomas. The 6-month PFS and overall survivals were $55 \%$ and $79 \%$, respectively. At least a partial response was observed in $61 \%$ of patients, and dose of dexamethasone was decreased in $67 \%$. These findings indicate that bevacizumab and irinotecan can be an active regimen for recurrent anaplastic gliomas.

3.4. Additional Treatment to Bevacizumab for Recurrent Malignant Gliomas. The addition of targeting therapy or radiotherapy to bevacizumab has been performed for recurrent malignant gliomas. Gutin et al. retrospectively analyzed bevacizumab and stereotactic radiotherapy (30 Gy in 5 fractions) for 25 recurrent malignant gliomas [30]. There were 20 glioblastomas and 5 anaplastic gliomas in this study with all patients receiving prior radiotherapy. Response rate was $50 \%$, and 6-month PFS was $65 \%$ in glioblastoma patients. Three patients (12\%) discontinued treatment due to tumor hemorrhage, wound dehiscence, and bowel perforation, although no radiation necrosis was detected. The authors concluded that treatment was well tolerated and beneficial for recurrent malignant gliomas. Cuneo et al. retrospectively evaluated the efficacy and safety of stereotactic radiosurgery and adjuvant bevacizumab for recurrent malignant gliomas [31]. Median PFS was 5.2 months, and 1-year overall survival was $50 \%$ in glioblastoma patients treated by radiosurgery and adjuvant bevacizumab. These results were significantly better than radiosurgery and other drugs. The authors concluded that salvage radiosurgery and bevacizumab improve outcomes in recurrent malignant gliomas.

A phase II study of bevacizumab plus erlotinib, an epidermal growth factor receptor (EGFR) tyrosine kinase inhibitor, was performed for patients with recurrent malignant gliomas [32]. This study included 25 glioblastomas and 32 anaplastic gliomas. The response rate and 6-month PFS were 48\% and $28 \%$ for glioblastoma and $31 \%$ and $44 \%$ for anaplastic gliomas, respectively. Grade 1 or 2 rash, mucositis, diarrhea, and fatigue were the most common adverse events. The authors concluded that treatment was tolerated, but the additional benefits of erlotinib were unclear when compared with historical bevacizumab-containing regimens. These studies indicate that additional therapy to bevacizumab can be promising strategy, although it is still unclear which agent has the efficacy in combination with bevacizumab for malignant gliomas. Further studies are required to establish the additional agents to bevacizumab.

\section{Resistance to Bevacizumab}

In the maintenance of bevacizumab, patients with malignant gliomas inevitably experience tumor recurrence. Furthermore, recurrent tumors after bevacizumab failure are 
reported to be more aggressive with rebound edema [38]. Although additional agents to bevacizumab have been attempted for patients after bevacizumab failure, disease prognosis was extremely poor with median PFS of 37.5 days and 6-month PFS of $2 \%$, respectively [39]. The authors concluded that alternative strategies should be considered for these patients. De Groot et al. showed that bevacizumab induced a particularly invasive tumor phenotype expressing insulin-like growth factor binding protein-2 and matrix metalloprotease-2 in glioblastoma [40].

Preclinical studies indicated that alternative pro-angiogenic signaling pathways are upregulated in resistance to antiangiogenic therapies [41]. These other angiogenic factors such as fibroblast growth factors and platelet-derived growth factors (PDGF) can compensate for the loss of VEGF activity under bevacizumab treatment [42]. Additional agents inhibiting other antiangiogenic pathways may suppress these resistances, and further clinical and animal studies are clearly required to overcome the resistance of bevacizumab.

\section{Imaging of Response to Antiangiogenic Therapy}

Most studies defined partial and complete responses as radiological objective response according to McDonald criteria that are based on contrast-enhanced CT or MRI [43]. However, an accurate assessment of tumor response by conventional modality is limited, since bevacizumab directly alters tumor blood vessels [44]. As a result of this, response rate and 6-month PFS are debatable as a measure of antitumor activity [44]. Norden et al. reported that bevacizumab suppressed enhancing tumor recurrence, but not nonenhancing and infiltrative tumor growth, indicating that bevacizumab may change the recurrence patterns of malignant gliomas [45]. Iwamoto et al. reported that contrast-enhanced MRI did not adequately evaluate disease status, whereas nonenhancing tumor recurrence was significantly associated with overall survival in recurrent glioblastoma treated by bevacizumab [46]. Given these findings, The Response Assessment in Neuro-Oncology Working Group was established to develop the new response criteria for clinical trials of brain tumors [47]. They proposed to incorporate $\mathrm{T} 2$ and fluid-attenuated inversion recovery (FLAIR) changes on MRI to assess the infiltrative pattern progression of malignant gliomas.

Other studies have looked into establishing a reliable radiological modality in antiangiogenesis therapy. Positron emission tomography (PET) using $\left[{ }^{18} \mathrm{~F}\right]$ fluorothymidine (FLT) offers noninvasive assessment of cell proliferation [48]. The response measured by FLT-PET significantly predicted the overall survival in recurrent glioblastoma treated by bevacizumab $(P=0.061)$ [49]. Recently, Ellingson et al. reported that relative nonenhancing tumor ratio, the ratio of FLAIR to contrast-enhancing volume, was predictive for overall survival and PFS in the treatment of bevacizumab for recurrent glioblastoma [50]. Further studies are warranted to establish the imaging modality to evaluate the response to antiangiogenic therapy and to predict the prognosis.

\section{Biological Markers Predicting Response}

A variety of biomarkers predicting the efficacy of bevacizumab have been reported in several tumor types including malignant gliomas [51]. These predictive biomarkers are expected to lead to a personalized therapy that selects patients who can benefit from bevacizumab. Sathornsumetee et al. examined several biological markers in recurrent malignant gliomas treated by bevacizumab and irinotecan [52]. High VEGF expression was significantly associated with higher radiographic response $(P=0.024)$, and high carbonic anhydrase 9 expression predicted poor overall survival $(P=0.016)$. Higher hypoxia-inducible factor-2 alpha and VEGF receptor-2 expressions were also reported to be associated with poor survival in recurrent malignant gliomas treated by bevacizumab and erlotinib [32].

Recently, circulating VEGF concentrations are reported to predict the prognosis in solid tumors treated by bevacizumab $[51,53]$. The measurement of circulating proteins is an attractive strategy since blood is easily accessible and the assay is inexpensive. Circulating VEGF concentrations are expected to reflect VEGF-dependent angiogenesis and to predict the benefit from bevacizumab [51]. Gururangan et al. examined the VEGFR-2 phosphorylation in peripheral blood mononuclear cells in recurrent malignant gliomas and diffuse brainstem glioma treated by bevacizumab [54]. They showed that circulating VEGFR-2 was inhibited by bevacizumab, but they did not show information on whether it is a prognostic biomarker. These clinical trials have provided some potential predictive markers (e.g. tumor VEGF expression or circulating markers), which require a phase III study for proper evaluation [55].

\section{Addition of Bevacizumab to Temozolomide and Radiotherapy for Newly Diagnosed Glioblastoma}

Several clinical studies have been performed to evaluate the safety and efficacy of the addition of bevacizumab for newly diagnosed glioblastoma (Table 1). Lai et al. reported a phase II study of the addition of bevacizumab to the standard treatment of temozolomide and radiotherapy for 70 newly diagnosed glioblastomas [14]. Bevacizumab was intravenously administered every 2 weeks from the first day of treatment. The median overall survival and PFS were 19.6 and 13.6 months, respectively. The authors concluded that the addition of bevacizumab improved PFS but not overall survival when compared with a control group treated with first-line temozolomide and radiotherapy who had mostly received bevacizumab at recurrence. Another phase II study also reported preliminary results on the addition of bevacizumab to the standard temozolomide and radiotherapy regimen in 125 newly diagnosed glioblastomas [15]. In this study, toxicity was minimal, and most patients (90\%) continued treatment, with median PFS of 13.8 months. Recently, Desjardins et al. reported a phase II study of bevacizumab in combination with temozolomide plus radiotherapy followed by bevacizumab, temozolomide, and irinotecan for 125 newly diagnosed glioblastomas at the 
Society for Neuro-Oncology (SNO) annual meeting in 2010 [33]. This study had median overall survival of 21.3 months and PFS of 13.8 months, respectively. These studies showed encouraging results; however, it is still unclear whether the addition of bevacizumab to standard temozolomide and radiotherapy can improve the overall survival.

Currently, two randomized phase III trials, ROTG 0825 and AVAGLIO, are ongoing for newly diagnosed glioblastoma treated by temozolomide and radiotherapy with or without bevacizumab $[56,57]$. These studies will show the role of bevacizumab in frontline treatment in glioblastoma patients.

\section{The Effect of Bevacizumab on Radiation Adverse Events}

Bevacizumab has been reported to affect the specific adverse events of radiotherapy [58, 59]. Sherman et al. reported that six glioblastoma patients developed severe radiation optic neuropathy following bevacizumab [58]. All of them received $60 \mathrm{~Gy}$ in 30 fractions in the initial treatment. Patients received a median of 7.5 doses of bevacizumab followed by onset of visual symptoms. Although the detailed mechanism remains unclear, the authors indicated that bevacizumab decreases optic nerve tolerance to radiation. Another case series study reported that bevacizumab induced optic neuropathy and Brown-Sequard syndrome after irradiation [59]. The authors hypothesized that bevacizumab following radiotherapy inhibits VEGF-dependent repair of normal neural tissue.

In contrast, bevacizumab has been reported to be effective for the management of radiation necrosis and retinopathy $[60,61]$. Radiation necrosis is a serious complication of radiotherapy and includes extended edema. Pathological findings show that endothelial cell dysfunction causes tissue hypoxia and necrosis with the local cytokine release, including VEGF $[62,63]$. Corticosteroids, surgery, anticoagulation, and hyperbaric oxygen have been performed, although there is no evidence to support routine use in clinical practice [64]. Retrospective studies have shown that bevacizumab decreased the edema and improved the clinical outcome in patients with radiation necrosis $[60,65-$ 67]. Interestingly, a small randomized trial was recently performed to demonstrate this effect [68]. Patients having radiation necrosis with progressive neurologic symptoms were assigned to bevacizumab $(n=14)$ and placebo groups $(n=7)$. Bevacizumab was intravenously administered every 3 weeks for 12 weeks. Radiological response and improvement of neurological symptoms were observed in the bevacizumab treated group but not in placebo group. The authors concluded that the class I evidence of bevacizumab efficacy for radiation necrosis was shown in this study.

Radiation retinopathy is a chronic and progressive condition that results from radiation exposure. Retinal vascular endothelial cell damage causes microaneurysms, telangiectasias, neovascularization, vitreous hemorrhage, macular edema, and tractional retinal detachment. Radiation retinopathy has been treated by laser photocoagulation, corticosteroids, and anticoagulation, although the management is still challenging [61]. Bevacizumab has been expected to be a therapeutic modality for radiation retinopathy. Finger reported that intravitreal injection of bevacizumab was effective for retinal hemorrhage, exudation, and edema, which improved visual acuity of patients [69]. There were no ocular and systemic side effects by bevacizumab. Furthermore, the authors recently showed that intravitreal bevacizumab was effective for radiation optic neuropathy [70].

Although these results indicate that some radiation vasculopathies are potentially treatable by bevacizumab, exacerbation of radiation necrosis by this drug was also reported [71]. It is still unclear how bevacizumab affects the radiation adverse events. Meticulous followup is required when bevacizumab is administered after radiotherapy. Animal models of radiation necrosis are needed to investigate the mechanism of bevacizumab.

\section{Other Antiangiogenic Drugs for Malignant Gliomas}

VEGF has been shown to be the main player in tumor angiogenesis, and its inhibitor, bevacizumab, has been thoroughly investigated in clinical and animal studies. Other drugs such as pan VEGF receptor tyrosine kinase inhibitors have also been reported to inhibit VEGF pathways. Several biological pathways including integrin, fibroblast growth factor, and PDGF also are associated with the angiogenesis. Currently, several types of antiangiogenic drugs have been investigated and used in clinical trials for recurrent as well as newly diagnosed glioblastoma [72]. In this section, we review these drugs and the results of clinical trials (Table 2).

9.1. Cilengitide. Cilengitide competitively binds $\alpha v \beta 3$ and $\alpha v \beta 5$ integrin receptors that are expressed on tumor cells and activated endothelial cells during angiogenesis. Cilengitide can directly inhibit the growth of integrin-expressing tumor cells and indirectly act as an antiangiogenesis agent $[87,88]$. Glioblastoma cells express integrin receptors, and cilengitide has shown an antitumor effect in glioblastoma xenografts in vivo $[89,90]$. A randomized phase II study of 81 recurrent glioblastoma was performed to determine the efficacy and safety of cilengitide [73]. The patients were randomly assigned to receive either 500 or $2000 \mathrm{mg}$ of cilengitide twice weekly. Patients treated with $2000 \mathrm{mg}$ showed a trend toward better results with 6-month PFS of $15 \%$. The treatment was well tolerated, and significant hematologic toxicity was uncommon. A phase I/IIa study of cilengitide combined with temozolomide and radiotherapy for 52 newly diagnosed glioblastoma patients was conducted [74]. This combination therapy was well tolerated without additional toxicity, and median overall survival was 16.1 months. The authors concluded that this regimen showed promising activity against newly diagnosed glioblastoma when compared with historical controls. Based on these results, two randomized trials, CENTRIC and CORE, are currently ongoing to determine the efficacy of cilengitide for newly diagnosed glioblastoma with or without a methylated 
TABLE 2: Other antiangiogenesis drugs for recurrent or newly diagnosed malignant gliomas.

\begin{tabular}{|c|c|c|c|c|c|c|c|c|}
\hline Target & Study & Agent & Patients & $\mathrm{RR}$ & MPFS & 6-PFS & MST & Ref. \\
\hline \multirow{2}{*}{ Integrin } & \multirow{2}{*}{ II } & Cilengitide (500 mg/day) & 41 recurrent GBM & $5 \%$ & 7.9 months & $10 \%$ & 6.5 months & \multirow[b]{2}{*}[73]{} \\
\hline & & (2000 mg/day) & 40 recurrent GBM & $13 \%$ & 8.1 months & $15 \%$ & 9.9 months & \\
\hline Integrin & I/IIa & Cilengitide + RT/TMZ & $\begin{array}{l}52 \text { Newly diagnosed } \\
\text { GBM }\end{array}$ & - & 8.0 months & $69 \%$ & 16.1 months & [74] \\
\hline bFGF & II & Thalidomide & 39 recurrent MG & $6 \%$ & 2.5 months & - & 7.0 months & {$[75]$} \\
\hline bFGF & II & Thalidomide + carmustine & 40 recurrent $\mathrm{MG}$ & $24 \%$ & 3.3 months & $28 \%$ & - & {$[76]$} \\
\hline bFGF & II & Thalidomide + irinotecan & 32 recurrent GBM & $6 \%$ & 3.3 months & $25 \%$ & 9.0 months & [77] \\
\hline bFGF & I & Lenalidomide & 24 recurrent GBM & $0 \%$ & 1.8 months & $13 \%$ & 6.0 months & [78] \\
\hline VEGFR & II & Cediranib (45 mg/day) & 31 recurrent $\mathrm{GBM}$ & $27 \%$ & 3.9 months & $26 \%$ & 7.6 months & {$[79]$} \\
\hline \multirow{3}{*}{ VEGFR } & \multirow{3}{*}{ III } & Cediranib (30 mg/day) & 325 recurrent GBM & - & - & $16 \%$ & - & \multirow{3}{*}[33]{} \\
\hline & & Cediranib (20 mg/day) + lomustine & & - & - & $35 \%$ & - & \\
\hline & & Lomustine + placebo & & - & - & $26 \%$ & - & \\
\hline VEGFR & II & Adjuvant sorafenib + TMZ & $\begin{array}{c}47 \text { newly diagnosed } \\
\text { GBM }\end{array}$ & $13 \%$ & 6.0 months & $50 \%$ & 12 months & {$[80]$} \\
\hline VEGFR & II & Sunitinib & 21 recurrent MG & $0 \%$ & 1.6 months & - & 3.8 months & {$[81]$} \\
\hline VEGFR & I & Vatalanib + RT/TMZ & $\begin{array}{l}19 \text { newly diagnosed } \\
\text { GBM }\end{array}$ & $13 \%$ & 7.2 months & - & 16.2 months & {$[82]$} \\
\hline VEGFR & II & Pazopanib & 35 recurrent GBM & $6 \%$ & 3.0 months & $3 \%$ & 8.8 months & [83] \\
\hline PDGFR & II & Imatinib & 31 recurrent GBM & $6 \%$ & 1.7 months & $16 \%$ & 5.2 months & [84] \\
\hline \multirow[b]{2}{*}{ PDGFR } & \multirow[b]{2}{*}{ III } & Imatinib & 120 recurrent GBM & - & 1.5 months & $7 \%$ & 5.3 months & \multirow[b]{2}{*}[85]{} \\
\hline & & Imatinib + hydroxyurea & 120 recurrent GBM & - & 1.5 months & $5 \%$ & 4.8 months & \\
\hline PDGFR & $\mathrm{R}$ & Dasatinib & 14 recurrent GBM & $0 \%$ & 0.9 months & $0 \%$ & 2.6 months & {$[86]$} \\
\hline
\end{tabular}

RR: response rate; MPFS: median progression-free survival; 6-PFS: 6-month progression-free survival; MST: median overall survival time; GBM: glioblastoma multiforme; RT: radiotherapy; TMZ: temozolomide; bFGF: basic fibroblast growth factor; MG: malignant gliomas; VEGFR: vascular endothelial growth factor receptor; PDGFR: platelet-derived growth factor receptor; R: retrospective.

$\mathrm{O}^{6}$-methylguanine-DNA methyltransferase (MGMT) promoter $[91,92]$.

9.2. Thalidomide and Lenalidomide. Thalidomide was developed as a sedative drug in 1950s and was withdrawn due to teratogenic effects. However, thalidomide was recently reported to have an antiangiogenic activity by inhibiting basic fibroblast growth factor (bFGF) [93], which can be exploited as an antitumor drug. Several clinical trials have been performed to assess the efficacy and safety of thalidomide for vascular tumors including malignant gliomas. This drug has since been approved by the FDA for the treatment of malignant myeloma [94]. Fine et al. showed a phase II study of thalidomide alone for 39 patients with recurrent malignant gliomas [75]. Thalidomide was well tolerated with modest sedation and constipation, although median PFS and overall survival were 2.5 months and 7.0 months, respectively. Another phase II study of thalidomide combined with carmustine was performed for 40 recurrent malignant gliomas [76]. Although the addition of carmustine seemed to improve the prognosis, the response rate and median PFS of combination group were $24 \%$ and 3.3 months, respectively. Puduvalli et al. reported a phase II trial of thalidomide and irinotecan for 32 recurrent glioblastomas [77]. The combination therapy was well tolerated with mild myelosuppression and sedation. At least a partial response was detected in two patients (6\%), and 6-month PFS was $25 \%$, respectively. These results indicate that thalidomide plus cytotoxic agents seem to have a mild antitumor activity for recurrent malignant gliomas patients when compared with thalidomide alone.

Lenalidomide, a potent structural and functional thalidomide analog, has antiangiogenic, anti-inflammatory, and immunomodulatory activities in preclinical studies $[95,96]$. This drug is approved by the FDA for myelodysplastic syndrome with chromosome $5 \mathrm{q}$ deletion and multiple myeloma. Recently, lenalidomide has been performed for recurrent brain tumors in clinical trials $[78,97]$. Fine et al. reported that lenalidomide was well tolerated; however, no objective responses were seen in a phase I study [78]. Median 6-month PFS was $12.5 \%$ in recurrent glioblastoma patients. Warren et al. conducted a phase I study of lenalidomide for pediatric patients with recurrent or progressive brain tumors [97]. This treatment was well tolerated with the primary toxicity being myelosuppression. Partial responses were seen in two patients (4\%) with low-grade gliomas. Because these studies were phase I trials, further investigations are required to evaluate the antitumor activity for malignant gliomas.

9.3. VEGF Receptor Tyrosine Kinase Inhibitors (Cediranib, Sorafenib, Sunitinib, Vatalanib, and Pazopanib). Currently, VEGF receptor tyrosine kinase inhibitors are viewed as 
promising antiangiogenic agents in the setting of malignant gliomas. Cediranib was developed as an oral pan-VEGF receptor tyrosine kinase inhibitor. Preclinical studies showed that cediranib normalized tumor vasculature and decreased the edema in glioblastoma, improving the prognosis without inhibition of tumor growth [98, 99]. Batchelor et al. conducted a phase II study of cediranib for 31 recurrent glioblastoma patients [79]. Patients were administered a $45 \mathrm{mg} /$ day dose of cediranib. Partial response according to the MacDonald criteria was observed in $26.6 \%$ of patients, and 6-month PFS was $25.8 \%$. Corticosteroids were reduced or discontinued in $27 \%$ of patients. Toxicities were manageable, and common Grade 3 to 4 toxicities were fatigue, hypertension, and diarrhea. Furthermore, they showed the changes of growth factors in plasma after cediranib (e.g., bFGF, VEGF receptor 1, and matrix metalloproteinase-2), which were associated with treatment response or survival in this therapy. Based on these promising results, the authors conducted a phase III study of cediranib for 325 patients with recurrent glioblastoma, and the preliminary results were reported at the 2010 SNO annual meeting [33]. Patients were assigned on a $2: 2: 1$ ratio to cediranib monotherapy $30 \mathrm{mg}$ /day, combination of cediranib $20 \mathrm{mg}$ /day plus lomustine, and lomustine monotherapy plus placebo groups. The 6-month PFS was $16 \%$ in cediranib monotherapy, $34.5 \%$ in the combination, and $25.8 \%$ in lomustine plus placebo groups, respectively, although the results were not significantly different between these groups. The efficacy of cediranib monotherapy seems to be less than the initial phase II study, and the possible reason for this discrepancy is that different doses of cediranib were used between two studies.

Sorafenib and sunitinib are inhibitors of multiple receptor tyrosine kinases including VEGF receptor. Sorafenib was approved by the FDA for the treatment of advanced renal cell carcinoma and hepatocellular carcinoma [100]. Hainsworth et al. conducted a phase II trial of concurrent radiotherapy and temozolomide followed by adjuvant sorafenib and temozolomide for 47 newly diagnosed glioblastomas [80]. This regimen was well tolerated without significant grade 3 or 4 toxicities, although median overall survival and PFS were 12 months and 6 months, respectively. The authors concluded that the addition of sorafenib did not appear to improve the prognosis of these patients. Sunitinib was reported in a phase II study of 21 recurrent malignant gliomas [81]. No objective responses were detected, and median overall survival and PFS were 3.8 and 1.6 months, respectively. This study showed that single-agent sunitinib had insufficient activity for recurrent malignant gliomas.

Vatalanib is a small molecule inhibitor of VEGF receptor, PDGF receptor, and c-kit. In a phase I trial, vatalanib was added to the standard regimen of temozolomide and radiotherapy for 19 newly diagnosed glioblastomas [82]. Response rate was $13 \%$, and median overall survival was 16.2 months, respectively. Pazopanib is a multitargeted tyrosine kinase inhibitor, including VEGF receptor-1, -2 , and -3. A phase II trial of pazopanib was performed for recurrent glioblastoma [83]. However, this drug did not have enough antitumor activity with response rate of $5.7 \%$ and median PFS of 3.0 months.
Despite several trials of VEGF receptor tyrosine kinase inhibitors, the efficacy has not been established. In a retrospective study of glioblastoma patients who failed VEGF receptor tyrosine kinase inhibitors, bevacizumab salvage therapy still provided benefits with response rate of $21 \%$ and 6-month PFS of $12.5 \%$, respectively [101]. Although there are no comparative studies, VEGF receptor inhibition therapy may be less effective for malignant gliomas when compared with bevacizumab [102].

9.4. PDGF Receptor Tyrosine Kinase Inhibitor (Imatinib, Dasatinib, and Tandutinib). The PDGF pathway also plays a role in angiogenesis [103]. PDGF receptor inhibitors (e.g., imatinib, dasatinib, and tandutinib) have been performed in clinical trials of malignant gliomas. Imatinib is a multitargeted tyrosine kinase inhibitor and blocks PDGF receptor $\alpha$, PDGF receptor $\beta$, and c-KIT receptor. Preclinical study has demonstrated the antitumor effect of imatinib on glioblastoma cell lines [104]. A phase II study of imatinib was performed for 112 recurrent gliomas [84]. The 6-month PFS was $16 \%$ in glioblastoma, $4.0 \%$ in pure/mixed anaplastic oligodendrogliomas, and $9 \%$ in low-grade or anaplastic astrocytoma. In 31 glioblastoma patients, response rate was $6 \%$, and median survival was 5.2 months, respectively. This study indicated that single agent imatinib was well tolerated but had limited antitumor activity. A randomized phase III study was conducted for 240 recurrent glioblastoma patients treated by hydroxyurea with or without imatinib [85]. The results from the two arms were very similar, and 6-month PFS was $5 \%$ in the combination arm and $7 \%$ in the imatinib alone arm, respectively. The authors concluded that there were no clinical benefits from the addition of imatinib. Taken together, these results suggest that imatinib is discouraged in recurrent glioblastoma patients.

Dasatinib and tandutinib are oral molecule inhibitors of several targets, including PDGF and c-kit. Dasatinib was approved by FDA for the treatment of chronic myelogenous leukemia [105]. A retrospective study reported the efficacy of dasatinib for 14 recurrent glioblastomas who failed bevacizumab therapy [86]. However, objective response rate was $0 \%$, and 6-month PFS was $0 \%$, respectively. Currently, a phase II trial of dasatinib (RTOG 0627) is ongoing to evaluate the efficacy and safety for recurrent glioblastoma or gliosarcoma [106]. Combined treatments with tandutinib and bevacizumab are being performed in a phase II study for recurrent malignant gliomas [107]. Preliminary results cautioned that neuromuscular junction dysfunction was observed in this regimen.

Although PDGF receptor inhibitors are effective in preclinical studies, it is still unclear whether these drugs have an antitumor effect in malignant glioma patients. One possible reason for the limited antitumor effect is that PDGF receptor inhibitor such as imatinib cannot cross the bloodbrain barrier via the P-glycoprotein efflux pump [108].

\section{Summary and Perspectives}

Despite advances in treatment therapeutics, patients with malignant gliomas still have poor prognosis. A better 
understanding of tumor angiogenesis has allowed us to target VEGF in antiangiogenic therapy. Bevacizumab is considered as a well-established antiangiogenic therapy in several solid tumors. A phase II trials of recurrent glioblastoma showed favorable response rates $(28 \%$ to $57 \%)$ and 6-month PFS (29\% to 50.3\%) [11-13]. Based on these promising results, bevacizumab was approved by the FDA for the recurrent glioblastoma. Regarding recurrent anaplastic gliomas, bevacizumab has been reported to be effective as well $[28,29]$. Additional therapies (e.g., chemotherapy, targeting therapy, and radiotherapy) to bevacizumab have been reported for recurrent malignant gliomas, and these results were encouraging. However, the timing, dosing, and the ideal treatment partners of bevacizumab have remained controversial. Further investigations are warranted to establish an antiangiogenic treatment for recurrent malignant gliomas.

Bevacizumab is expected to be on the frontline treatment of patients with glioblastoma. Phase II trials have reported the addition of bevacizumab to standard temozolomide and radiotherapy regimen for newly diagnosed glioblastoma $[14,15]$. However, the authors concluded that this regimen improved PFS but not overall survival when compared with control group [14]. Currently two randomized phase III trials, RTOG 0825 and AVAGLIO, are ongoing to demonstrate the efficacy and safety of combined therapy of bevacizumab, temozolomide, and radiotherapy for newly diagnosed glioblastoma [56, 57]. These studies will show the role of bevacizumab in the first-line treatment of newly diagnosed glioblastoma.

Many other antiangiogenic therapies (e.g., cilengitide and cediranib) have also been performed in clinical trials. These studies showed encouraging results and are expected to improve the prognosis of malignant gliomas. However, some phase II trials have several limitations such as small sample size, possible enrollment bias, patient selection, and reliance on historical control data. These limitations are associated with a high false-positive rate, and the results from phase II studies are often not validated in phase III studies [109]. Phase II studies must be appropriately planned to have the greatest potential for informing the design of phase III trials [109].

Antiangiogenic therapies provide favorable results and seem to be attractive strategy in malignant gliomas. However, several problems such as including severe toxicities, resistance, evaluation of response, and lack of predictive biomarkers still remain. The unique severe adverse effects related to bevacizumab have been reported, such as CNS hemorrhage, deep venous thrombosis, and pulmonary emboli [11-13]. BRAIN study reported that Grade $\geq 3$ adverse events were observed $65.8 \%$ and $46.4 \%$ in bevacizumab plus irinotecan and bevacizumab alone groups, respectively [13]. To reduce the serious adverse events associated with bevacizumab, selecting appropriate patients, early assessment of toxicity, and adequate management should be required.

Malignant glioma patients maintained on bevacizumab inevitably experience the treatment failure. Recurrent tumors following bevacizumab failure appear to be more aggressive with rebound edema [38]. Preclinical study showed that other angiogenic factors, such as fibroblast growth factors and PDGF, can compensate for the loss of VEGF activity under bevacizumab treatment [42]. A novel therapeutic strategy is required to overcome the resistance to bevacizumab of malignant gliomas.

An accurate assessment of tumor response by conventional modality is limited in antiangiogenic therapy due to alterations in tumor blood vessels [44]. The Response Assessment in Neuro-Oncology Working Group proposed that T2 and FLAIR changes on MRI should include the response criteria [47]. FDG-FLT is also expected to accurately evaluate the treatment response in bevacizumab due to its ability to detect cell proliferation [48].

Tumor VEGF expressions or circulating markers potentially predict the prognosis in malignant glioma treated by bevacizumab, although the rational biomarker has not been established. Novel biological markers are required to investigate, providing a personalized treatment that selects the patients who can benefit from bevacizumab.

Although several limitations on antiangiogenic therapy have been reported, this treatment is expected to improve the prognosis of malignant gliomas. Further investigation is warranted to establish the safe and effective antiangiogenic therapy for malignant gliomas.

\section{References}

[1] P. Y. Wen and S. Kesari, "Malignant gliomas in adults," The New England Journal of Medicine, vol. 359, no. 5, pp. 492507, 2008.

[2] R. Stupp, W. P. Mason, M. J. van den Bent et al., "Radiotherapy plus concomitant and adjuvant temozolomide for glioblastoma," The New England Journal of Medicine, vol. 352, no. 10, pp. 987-996, 2005.

[3] M. E. Hegi, A. C. Diserens, T. Gorlia et al., "MGMT gene silencing and benefit from temozolomide in glioblastoma," The New England Journal of Medicine, vol. 352, no. 10, pp. 997-1003, 2005.

[4] R. Stupp, M. E. Hegi, W. P. Mason et al., "Effects of radiotherapy with concomitant and adjuvant temozolomide versus radiotherapy alone on survival in glioblastoma in a randomised phase III study: 5-year analysis of the EORTCNCIC trial," The Lancet Oncology, vol. 10, no. 5, pp. 459-466, 2009.

[5] W. J. Curran Jr., C. B. Scott, J. Horton et al., "Recursive partitioning analysis of prognostic factors in three radiation therapy oncology group malignant glioma trials," Journal of the National Cancer Institute, vol. 85, no. 9, pp. 704-710, 1993.

[6] M. D. Prados, W. Seiferheld, H. M. Sandler et al., "Phase III randomized study of radiotherapy plus procarbazine, lomustine, and vincristine with or without BUdR for treatment of anaplastic astrocytoma: final report of RTOG 9404," International Journal of Radiation Oncology Biology Physics, vol. 58, no. 4, pp. 1147-1152, 2004.

[7] E. T. Wong, K. R. Hess, M. J. Gleason et al., "Outcomes and prognostic factors in recurrent glioma patients enrolled onto phase II clinical trials," Journal of Clinical Oncology, vol. 17, no. 8, pp. 2572-2578, 1999. 
[8] D. N. Louis, H. Ohgaki, O. D. Wiestler, and W. K. Cavenee, Eds., WHO Classification of Tumours of the Central Nervous System, IARC Press, Lyon, France, 4th edition, 2007.

[9] J. C. Reijneveld, E. E. Voest, and M. J. B. Taphoorn, "Angiogenesis in malignant primary and metastatic brain tumors," Journal of Neurology, vol. 247, no. 8, pp. 597-608, 2000.

[10] N. Ferrara, H. P. Gerber, and J. LeCouter, "The biology of VEGF and its receptors," Nature Medicine, vol. 9, no. 6, pp. 669-676, 2003.

[11] J. J. Vredenburgh, A. Desjardins, J. E. Herndon et al., "Bevacizumab plus irinotecan in recurrent glioblastoma multiforme," Journal of Clinical Oncology, vol. 25, no. 30, pp. 4722-4729, 2007.

[12] H. S. Friedman, M. D. Prados, P. Y. Wen et al., "Bevacizumab alone and in combination with irinotecan in recurrent glioblastoma," Journal of Clinical Oncology, vol. 27, no. 28, pp. 4733-4740, 2009.

[13] T. N. Kreisl, L. Kim, K. Moore et al., "Phase II trial of singleagent bevacizumab followed by bevacizumab plus irinotecan at tumor progression in recurrent glioblastoma," Journal of Clinical Oncology, vol. 27, no. 5, pp. 740-745, 2009.

[14] A. Lai, A. Tran, P. L. Nghiemphu et al., "Phase II study of bevacizumab plus temozolomide during and after radiation therapy for patients with newly diagnosed glioblastoma multiforme," Journal of Clinical Oncology, vol. 29, no. 2, pp. 142-148, 2011.

[15] J. J. Vredenburgh, A. Desjardins, J. P. Kirkpatrick et al., "Addition of bevacizumab to standard radiation therapy and daily temozolomide is associated with minimal toxicity in newly diagnosed glioblastoma multiforme," International Journal of Radiation Oncology, Biology, Physics. In press.

[16] M. S. Ahluwalia and C. L. Gladson, "Progress on antiangiogenic therapy for patients with malignant glioma," Journal of Oncology, vol. 2010, Article ID 689018, 14 pages, 2010.

[17] K. H. Plate, G. Breier, H. A. Weich, and W. Risau, "Vascular endothelial growth factor is a potential tumour angiogenssis factor in human gliomas in vivo," Nature, vol. 359, no. 6398, pp. 845-848, 1992.

[18] B. Millauer, L. K. Shawver, K. H. Plate, W. Risau, and A. Ullrich, "Glioblastoma growth inhibited in vivo by a dominant-negative Flk-1 mutant," Nature, vol. 367, no. 6463, pp. 576-578, 1994.

[19] Y. H. Zhou, F. Tan, K. R. Hess, and W. K. A. Yung, "The expression of PAX6, PTEN, vascular endothelial growth factor, and epidermal growth factor receptor in gliomas: relationship to tumor grade and survival," Clinical Cancer Research, vol. 9, no. 9, pp. 3369-3375, 2003.

[20] I. H. Chaudhry, D. G. O’Donovan, P. E. C. Brenchley, H. Reid, and I. S. D. Roberts, "Vascular endothelial growth factor expression correlates with tumour grade and vascularity in gliomas," Histopathology, vol. 39, no. 4, pp. 409-415, 2001.

[21] D. F. Stefanik, W. K. Fellows, L. R. Rizkalla et al., "Monoclonal antibodies to vascular endothelial growth factor (VEGF) and the VEGF receptor, FLT-1, inhibit the growth of C6 glioma in a mouse xenograt," Journal of Neuro-Oncology, vol. 55, no. 2, pp. 91-100, 2001.

[22] S. Bao, Q. Wu, S. Sathornsumetee et al., "Stem cell-like glioma cells promote tumor angiogenesis through vascular endothelial growth factor," Cancer Research, vol. 66, no. 16, pp. 7843-7848, 2006.
[23] F. Winkler, S. V. Kozin, R. T. Tong et al., "Kinetics of vascular normalization by VEGFR2 blockade governs brain tumor response to radiation: role of oxygenation, angiopoietin-1, and matrix metalloproteinases," Cancer Cell, vol. 6, no. 6, pp. 553-563, 2004.

[24] P. Carmeliet and R. K. Jain, "Angiogenesis in cancer and other diseases," Nature, vol. 407, no. 6801, pp. 249-257, 2000.

[25] N. Ferrara, K. J. Hillan, H. P. Gerber, and W. Novotny, "Discovery and development of bevacizumab, an anti-VEGF antibody for treating cancer," Nature Reviews Drug Discovery, vol. 3, no. 5, pp. 391-400, 2004.

[26] L. G. Presta, H. Chen, S. J. O'Connor et al., "Humanization of an anti-vascular endothelial growth factor monoclonal antibody for the therapy of solid tumors and other disorders," Cancer Research, vol. 57, no. 20, pp. 4593-4599, 1997.

[27] F. Kazazi-Hyseni, J. H. Beijnen, and J. H. M. Schellens, "Bevacizumab," Oncologist, vol. 15, no. 8, pp. 819-825, 2010.

[28] J. J. Vredenburgh, A. Desjardins, J. E. Herndon et al., "Phase II trial of bevacizumab and irinotecan in recurrent malignant glioma," Clinical Cancer Research, vol. 13, no. 4, pp. 12531259, 2007.

[29] A. Desjardins, D. A. Reardon, J. E. Herndon et al., "Bevacizumab plus irinotecan in recurrent WHO grade 3 malignant gliomas," Clinical Cancer Research, vol. 14, no. 21, pp. 7068-7073, 2008.

[30] P. H. Gutin, F. M. Iwamoto, K. Beal et al., "Safety and efficacy of bevacizumab with hypofractionated stereotactic irradiation for recurrent malignant gliomas," International Journal of Radiation Oncology Biology Physics, vol. 75, no. 1, pp. 156-163, 2009.

[31] K. C. Cuneo, J. J. Vredenburgh, J. H. Sampson et al., "Safety and efficacy of stereotactic radiosurgery and adjuvant bevacizumab in patients with recurrent malignant gliomas," International Journal of Radiation Oncology, Biology, Physics. In press.

[32] S. Sathornsumetee, A. Desjardins, J. J. Vredenburgh et al., "Phase II trial of bevacizumab and erlotinib in patients with recurrent malignant glioma," Neuro-Oncology, vol. 12, no. 12, pp. 1300-1310, 2010.

[33] M. S. Ahluwalia, "2010 Society for neuro-oncology annual meeting: a report of selected studies," Expert Review of Anticancer Therapy, vol. 11, no. 2, pp. 161-163, 2011.

[34] S. M. Gressett and S. R. Shah, "Intricacies of bevacizumabinduced toxicities and their management," Annals of Pharmacotherapy, vol. 43, no. 3, pp. 490-501, 2009.

[35] T. J. Semrad, R. O'Donnell, T. Wun et al., "Epidemiology of venous thromboembolism in 9489 patients with malignant glioma," Journal of Neurosurgery, vol. 106, no. 4, pp. 601-608, 2007.

[36] V. Ranpura, S. Hapani, and S. Wu, "Treatment-related mortality with bevacizumab in cancer patients: a meta-analysis," JAMA-Journal of the American Medical Association, vol. 305, no. 5, pp. 487-494, 2011.

[37] J. C. Buckner, "Factors influencing survival in high-grade gliomas," Seminars in Oncology, vol. 30, no. 6, pp. 10-14, 2003.

[38] S. Ananthnarayan, J. Bahng, J. Roring et al., "Time course of imaging changes of GBM during extended bevacizumab treatment," Journal of Neuro-Oncology, vol. 88, no. 3, pp. 339-347, 2008.

[39] E. C. Quant, A. D. Norden, J. Drappatz et al., "Role of a second chemotherapy in recurrent malignant glioma patients who progress on bevacizumab," Neuro-Oncology, vol. 11, no. 5, pp. 550-555, 2009. 
[40] J. F. de Groot, G. Fuller, A. J. Kumar et al., "Tumor invasion after treatment of glioblastoma with bevacizumab: radiographic and pathologic correlation in humans and mice," Neuro-Oncology, vol. 12, no. 3, pp. 233-242, 2010.

[41] G. Bergers and D. Hanahan, "Modes of resistance to antiangiogenic therapy," Nature Reviews Cancer, vol. 8, no. 8, pp. 592-603, 2008.

[42] J. M. L. Ebos, C. R. Lee, and R. S. Kerbel, "Tumor and hostmediated pathways of resistance and disease progression in response to antiangiogenic therapy," Clinical Cancer Research, vol. 15, no. 16, pp. 5020-5025, 2009.

[43] D. R. Macdonald, T. L. Cascino, S. C. Schold, and J. G. Cairncross, "Response criteria for phase II studies of supratentorial malignant glioma," Journal of Clinical Oncology, vol. 8, no. 7, pp. 1277-1280, 1990.

[44] A. S. Chi, A. D. Norden, and P. Y. Wen, "Antiangiogenic strategies for treatment of malignant gliomas," Neurotherapeutics, vol. 6, no. 3, pp. 513-526, 2009.

[45] A. D. Norden, G. S. Young, K. Setayesh et al., "Bevacizumab for recurrent malignant gliomas: efficacy, toxicity, and patterns of recurrence," Neurology, vol. 70, no. 10, pp. 779-787, 2008.

[46] F. M. Iwamoto, L. E. Abrey, K. Beal et al., "Patterns of relapse and prognosis after bevacizumab failure in recurrent glioblastoma," Neurology, vol. 73, no. 15, pp. 1200-1206, 2009.

[47] P. Y. Wen, D. R. Macdonald, D. A. Reardon et al., "Updated response assessment criteria for high-grade gliomas: response assessment in neuro-oncology working group," Journal of Clinical Oncology, vol. 28, no. 11, pp. 19631972, 2010.

[48] T. Barwick, B. Bencherif, J. M. Mountz, and N. Avril, "Molecular PET and PET/CT imaging of tumour cell proliferation using F-18 fluoro-L-thymidine: a comprehensive evaluation," Nuclear Medicine Communications, vol. 30, no. 12, pp. 908917, 2009.

[49] W. Chen, S. Delaloye, D. H. S. Silverman et al., "Predicting treatment response of malignant gliomas to bevacizumab and irinotecan by imaging proliferation with [18F] fluorothymidine positron emission tomography: a pilot study," Journal of Clinical Oncology, vol. 25, no. 30, pp. 4714-4721, 2007.

[50] B. M. Ellingson, T. F. Cloughesy, A. Lai, P. L. Nghiemphu, P. S. Mischel, and W. B. Pope, "Quantitative volumetric analysis of conventional MRI response in recurrent glioblastoma treated with bevacizumab," Neuro-Oncology, vol. 13, no. 4, pp. 401409, 2011.

[51] A. M. Jubb and A. L. Harris, "Biomarkers to predict the clinical efficacy of bevacizumab in cancer," The Lancet Oncology, vol. 11, no. 12, pp. 1172-1183, 2010.

[52] S. Sathornsumetee, Y. Cao, J. E. Marcello et al., "Tumor angiogenic and hypoxic profiles predict radiographic response and survival in malignant astrocytoma patients treated with bevacizumab and irinotecan," Journal of Clinical Oncology, vol. 26, no. 2, pp. 271-278, 2008.

[53] E. C. D. S. Pau, F. C. Arias, E. C. Pelaez et al., "Vascular endothelial growth factor (VEGF) serum levels are associated with survival in early stages of lung cancer patients," Cancer Investigation, vol. 28, no. 4, pp. 393-398, 2010.

[54] S. Gururangan, S. N. Chi, T. Young Poussaint et al., "Lack of efficacy of bevacizumab plus irinotecan in children with recurrent malignant glioma and diffuse brainstem glioma: a Pediatric Brain Tumor Consortium study," Journal of Clinical Oncology, vol. 28, no. 18, pp. 3069-3075, 2010.
[55] D. J. Sargent, B. A. Conley, C. Allegra, and L. Collette, "Clinical trial designs for predictive marker validation in cancer treatment trials," Journal of Clinical Oncology, vol. 23, no. 9, pp. 2020-2027, 2005.

[56] http://clinicaltrials.gov/ct2/show/NCT00884741?term=rtog +0825 \&rank $=1$.

[57] http://www.isesd.cv.ic.ac.uk.

[58] J. H. Sherman, D. G. Aregawi, A. Lai et al., "Optic neuropathy in patients with glioblastoma receiving bevacizumab," Neurology, vol. 73, no. 22, pp. 1924-1926, 2009.

[59] P. J. Kelly, M. J. Dinkin, J. Drappatz, K. N. O’Regan, and S. E. Weiss, "Unexpected late radiation neurotoxicity following bevacizumab use: a case series," Journal of Neuro-Oncology, vol. 102, no. 3, pp. 485-490, 2010.

[60] J. Gonzalez, A. J. Kumar, C. A. Conrad, and V. A. Levin, "Effect of bevacizumab on radiation necrosis of the brain," International Journal of Radiation Oncology Biology Physics, vol. 67, no. 2, pp. 323-326, 2007.

[61] G. P. Giuliari, A. Sadaka, D. M. Hinkle, and E. R. Simpson, "Current treatments for radiation retinopathy," Acta Oncologica, vol. 50, no. 1, pp. 6-13, 2011.

[62] J. R. Crossen, D. Garwood, E. Glatstein, and E. A. Neuwelt, "Neurobehavioral sequelae of cranial irradiation in adults: a review of radiation-induced encephalopathy," Journal of Clinical Oncology, vol. 12, no. 3, pp. 627-642, 1994.

[63] Y. Q. Li, J. R. Ballinger, R. A. Nordal, Z. F. Su, and C. S. Wong, "Hypoxia in radiation-induced blood-spinal cord barrier breakdown," Cancer Research, vol. 61, no. 8, pp. 33483354, 2001.

[64] P. Giglio and M. R. Gilbert, "Cerebral radiation necrosis," Neurologist, vol. 9, no. 4, pp. 180-188, 2003.

[65] E. T. Wong, M. Huberman, X. Q. Lu, and A. Mahadevan, "Bevacizumab reverses cerebral radiation necrosis," Journal of Clinical Oncology, vol. 26, no. 34, pp. 5649-5650, 2008.

[66] R. Torcuator, R. Zuniga, Y. S. Mohan et al., "Initial experience with bevacizumab treatment for biopsy confirmed cerebral radiation necrosis," Journal of Neuro-Oncology, vol. 94, no. 1, pp. 63-68, 2009.

[67] M. Furuse, S. Kawabata, T. Kuroiwa, and S. I. Miyatake, "Repeated treatments with bevacizumab for recurrent radiation necrosis in patients with malignant brain tumors: a report of 2 cases," Journal of Neuro-Oncology, pp. 1-5, 2010.

[68] V. A. Levin, L. Bidaut, P. Hou et al., "Randomized doubleblind placebo-controlled trial of bevacizumab therapy for radiation necrosis of the central nervous system," International Journal of Radiation Oncology, Biology, Physics, vol. 79, no. 5, pp. 1487-1495, 2010.

[69] P. T. Finger, "Radiation retinopathy is treatable with antivascular endothelial growth factor bevacizumab (Avastin)," International Journal of Radiation Oncology Biology Physics, vol. 70, no. 4, pp. 974-977, 2008.

[70] P. T. Finger and K. J. Chin, "Antivascular endothelial growth factor bevacizumab for radiation optic neuropathy: secondary to plaque radiotherapy," International Journal of Radiation Oncology, Biology, Physics. In press.

[71] D. S. Jeyaretna, W. T. Curry Jr., T. T. Batchelor, A. StemmerRachamimov, and S. R. Plotkin, "Exacerbation of cerebral radiation necrosis by bevacizumab," Journal of Clinical Oncology, vol. 29, no. 7, pp. e159-e162, 2011.

[72] K. Beal, L. E. Abrey, and P. H. Gutin, "Antiangiogenic agents in the treatment of recurrent or newly diagnosed glioblastoma: analysis of single-agent and combined modality approaches," Radiation Oncology, vol. 6, article 2, 2011. 
[73] D. A. Reardon, K. L. Fink, T. Mikkelsen et al., "Randomized phase II study of cilengitide, an integrin-targeting arginineglycine-aspartic acid peptide, in recurrent glioblastoma multiforme," Journal of Clinical Oncology, vol. 26, no. 34, pp. 5610-5617, 2008.

[74] R. Stupp, M. E. Hegi, B. Neyns et al., "Phase I/IIa study of cilengitide and temozolomide with concomitant radiotherapy followed by cilengitide and temozolomide maintenance therapy in patients with newly diagnosed glioblastoma," Journal of Clinical Oncology, vol. 28, no. 16, pp. 2712-2718, 2010.

[75] H. A. Fine, W. D. Figg, K. Jaeckle et al., "Phase II trial of the antiangiogenic agent thalidomide in patients with recurrent high-grade gliomas," Journal of Clinical Oncology, vol. 18, no. 4, pp. 708-715, 2000.

[76] H. A. Fine, P. Y. Wen, E. A. Maher et al., "Phase II trial of thalidomide and carmustine for patients with recurrent highgrade gliomas," Journal of Clinical Oncology, vol. 21, no. 12, pp. 2299-2304, 2003.

[77] V. K. Puduvalli, P. Giglio, M. D. Groves et al., "Phase II trial of irinotecan and thalidomide in adults with recurrent glioblastoma multiforme," Neuro-Oncology, vol. 10, no. 2, pp. 216-222, 2008.

[78] H. A. Fine, L. Kim, P. S. Albert et al., "A phase I trial of lenalidomide in patients with recurrent primary central nervous system tumors," Clinical Cancer Research, vol. 13, no. 23, pp. 7101-7106, 2007.

[79] T. T. Batchelor, D. G. Duda, E. di Tomaso et al., "Phase II study of cediranib, an oral pan-vascular endothelial growth factor receptor tyrosine kinase inhibitor, in patients with recurrent glioblastoma," Journal of Clinical Oncology, vol. 28, no. 17 , pp. 2817-2823, 2010.

[80] J. D. Hainsworth, T. Ervin, E. Friedman et al., "Concurrent radiotherapy and temozolomide followed by temozolomide and sorafenib in the first-line treatment of patients with glioblastoma multiforme," Cancer, vol. 116, no. 15, pp. 36633669, 2010.

[81] B. Neyns, J. Sadones, C. Chaskis et al., "Phase II study of sunitinib malate in patients with recurrent high-grade glioma," Journal of Neuro-Oncology, pp. 1-11, 2010.

[82] E. R. Gerstner, A. F. Eichler, S. R. Plotkin et al., "Phase I trial with biomarker studies of vatalanib (PTK787) in patients with newly diagnosed glioblastoma treated with enzyme inducing anti-epileptic drugs and standard radiation and temozolomide," Journal of Neuro-Oncology, vol. 103, no. 2, pp. 325-332, 2010.

[83] F. M. Iwamoto, K. R. Lamborn, H. I. Robins et al., "Phase II trial of pazopanib (GW786034), an oral multi-targeted angiogenesis inhibitor, for adults with recurrent glioblastoma (North American Brain Tumor Consortium Study 06-02)," Neuro-Oncology, vol. 12, no. 8, pp. 855-861, 2010.

[84] E. Raymond, A. A. Brandes, C. Dittrich et al., "Phase II study of imatinib in patients with recurrent gliomas of various histologies: a European organisation for research and treatment of cancer brain tumor group study," Journal of Clinical Oncology, vol. 26, no. 28, pp. 4659-4665, 2008.

[85] G. Dresemann, M. Weller, M. A. Rosenthal et al., "Imatinib in combination with hydroxyurea versus hydroxyurea alone as oral therapy in patients with progressive pretreated glioblastoma resistant to standard dose temozolomide," Journal of Neuro-Oncology, vol. 96, no. 3, pp. 393-402, 2010.

[86] C. Lu-Emerson, A. D. Norden, J. Drappatz et al., "Retrospective study of dasatinib for recurrent glioblastoma after bevacizumab failure," Journal of Neuro-Oncology. In press.
[87] G. D. Maurer, I. Tritschler, B. Adams et al., "Cilengitide modulates attachment and viability of human glioma cells, but not sensitivity to irradiation or temozolomide in vitro," Neuro-Oncology, vol. 11, no. 6, pp. 747-756, 2009.

[88] R. E. Nisato, J. C. Tille, A. Jonczyk, S. L. Goodman, and M. S. Pepper, " $\alpha \mathrm{v} \beta 3$ and $\alpha \mathrm{v} \beta 5$ integrin antagonists inhibit angiogenesis in vitro," Angiogenesis, vol. 6, no. 2, pp. 105-119, 2003.

[89] O. Schnell, B. Krebs, E. Wagner et al., "Expression of integrin $\alpha \mathrm{v} \beta 3$ in gliomas correlates with tumor grade and is not restricted to tumor vasculature," Brain Pathology, vol. 18, no. 3, pp. 378-386, 2008.

[90] S. Yamada, X. Y. Bu, V. Khankaldyyan, I. Gonzales-Gomez, J. G. McComb, and W. E. Laug, "Effect of the angiogenesis inhibitor Cilengitide (EMD 121974) on glioblastoma growth in nude mice," Neurosurgery, vol. 59, no. 6, pp. 1304-1312, 2006.

[91] R. Stupp, M. van den Bent, S. Erridge et al., "Cilengitide in newly diagnosed glioblastoma with MGMT promoter methylation: protocol of a multicenter, randomized, openlabel, controlled phase III trial (CENTRIC)," Journal of Clinical Oncology, vol. 28, no. 15, 2010, abstract TPS152.

[92] L. Nabors, K. Fink, D. Reardon et al., "Cilengitide in patients with newly diagnosed glioblastoma multiforme and unmethylated MGMT gene promoter: protocol of a multicenter, randomized, open-label, controlled phase II study," Journal of Clinical Oncology, vol. 28, no. 15, 2010, abstract TPS151.

[93] R. J. D’Amato, M. S. Loughnan, E. Flynn, and J. Folkman, "Thalidomide is an inhibitor of angiogenesis," Proceedings of the National Academy of Sciences of the United States of America, vol. 91, no. 9, pp. 4082-4085, 1994.

[94] S. Singhal, J. Mehta, R. Desikan et al., "Antitumor activity of thalidomide in refractory multiple myeloma," The New England Journal of Medicine, vol. 341, no. 21, pp. 1565-1571, 1999.

[95] K. Dredge, R. Horsfall, S. P. Robinson et al., "Orally administered lenalidomide (CC-5013) is anti-angiogenic in vivo and inhibits endothelial cell migration and Akt phosphorylation in vitro," Microvascular Research, vol. 69, no. 1-2, pp. 56-63, 2005.

[96] D. Zhu, L. G. Corral, Y. W. Fleming, and B. Stein, "Immunomodulatory drugs Revlimid@ (lenalidomide) and CC-4047 induce apoptosis of both hematological and solid tumor cells through NK cell activation," Cancer Immunology, Immunotherapy, vol. 57, no. 12, pp. 1849-1859, 2008.

[97] K. E. Warren, S. Goldman, I. F. Pollack et al., "Phase I trial of lenalidomide in pediatric patients with recurrent, refractory, or progressive primary CNS tumors: pediatric Brain Tumor Consortium Study PBTC-018," Journal of Clinical Oncology, vol. 29, no. 3, pp. 324-329, 2011.

[98] T. T. Batchelor, A. G. Sorensen, E. di Tomaso et al., "AZD2171, a pan-VEGF receptor tyrosine kinase inhibitor, normalizes tumor vasculature and alleviates edema in glioblastoma patients," Cancer Cell, vol. 11, no. 1, pp. 83-95, 2007.

[99] W. S. Kamoun, C. D. Ley, C. T. Farrar et al., "Edema control by cediranib, a vascular endothelial growth factor receptortargeted kinase inhibitor, prolongs survival despite persistent brain tumor growth in mice," Journal of Clinical Oncology, vol. 27, no. 15, pp. 2542-2552, 2009.

[100] R. Iyer, G. Fetterly, A. Lugade, and Y. Thanavala, "Sorafenib: a clinical and pharmacologic review," Expert Opinion on Pharmacotherapy, vol. 11, no. 11, pp. 1945-1955, 2010. 
[101] B. J. Scott, E. C. Quant, M. B. McNamara, P. A. Ryg, T. T. Batchelor, and P. Y. Wen, "Bevacizumab salvage therapy following progression in high-grade glioma patients treated with VEGF receptor tyrosine kinase inhibitors," NeuroOncology, vol. 12, no. 6, pp. 603-607, 2010.

[102] E. T. Wong and S. Brem, "Taming glioblastoma by targeting angiogenesis: 3 Yyears later," Journal of Clinical Oncology, vol. 29, no. 2, pp. 124-126, 2011.

[103] A. D. Laird, P. Vajkoczy, L. K. Shawver et al., "SU6668 is a potent antiangiogenic and antitumor agent that induces regression of established tumors," Cancer Research, vol. 60, no. 15, pp. 4152-4160, 2000.

[104] D. Hägerstrand, G. Hesselager, S. Achterberg et al., "Characterization of an imatinib-sensitive subset of high-grade human glioma cultures," Oncogene, vol. 25, no. 35, pp. 49134922, 2006.

[105] M. Talpaz, N. P. Shah, H. Kantarjian et al., "Dasatinib in imatinib-resistant Philadelphia chromosome-positive leukemias," The New England Journal of Medicine, vol. 354, no. 24, pp. 2531-2541, 2006.

[106] http://clinicaltrials.gov/ct2/show/NCT00423735?term=RTOG+0627\&rank=1.

[107] T. J. Lehky, F. M. Iwamoto, T. N. Kreisl, M. K. Floeter, and H. A. Fine, "Neuromuscular junction toxicity with tandutinib induces a myasthenic-like syndrome," Neurology, vol. 76, no. 3, pp. 236-241, 2011.

[108] H. Dai, P. Marbach, M. Lemaire, M. Hayes, and W. F. Elmquist, "Distribution of STI-571 to the brain is limited by P-glycoprotein-mediated efflux," Journal of Pharmacology and Experimental Therapeutics, vol. 304, no. 3, pp. 10851092, 2003.

[109] S. A. Cannistra, "Phase II trials in journal of clinical oncology," Journal of Clinical Oncology, vol. 27, no. 19, pp. 3073-3076, 2009. 


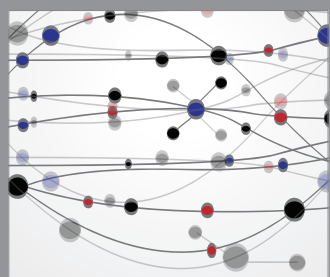

The Scientific World Journal
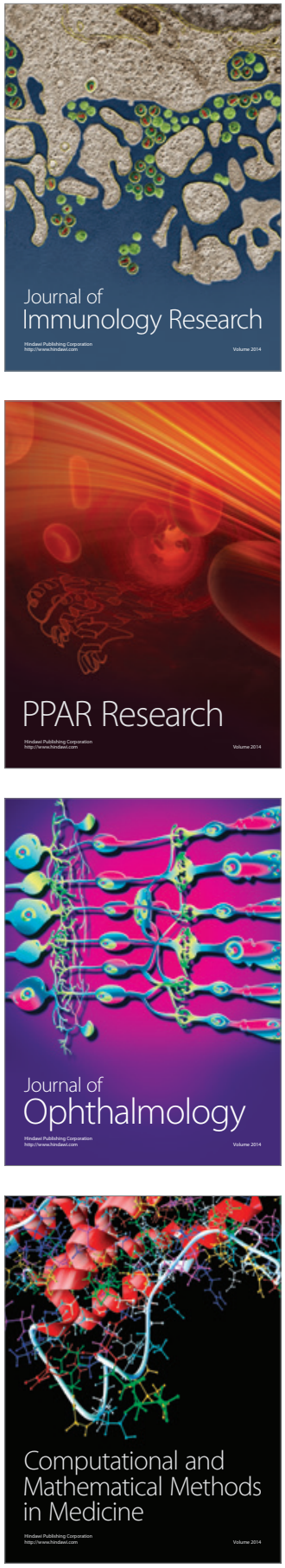

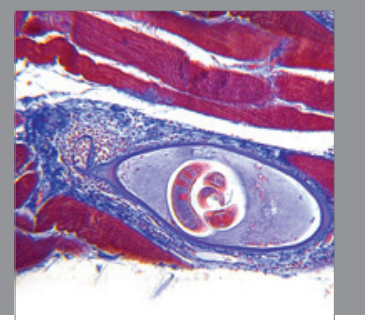

Gastroenterology

Research and Practice
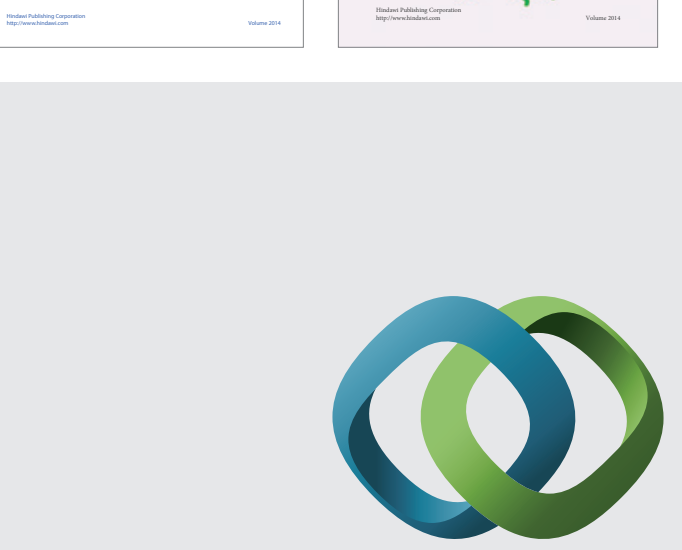

\section{Hindawi}

Submit your manuscripts at

http://www.hindawi.com
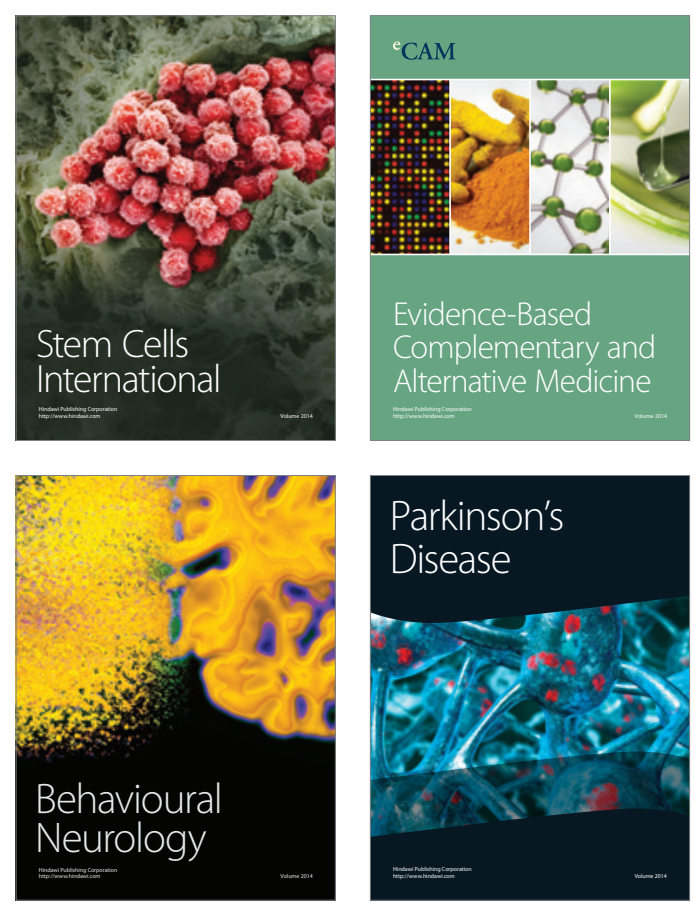

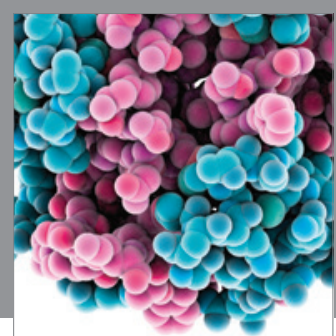

Journal of
Diabetes Research

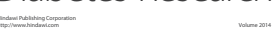

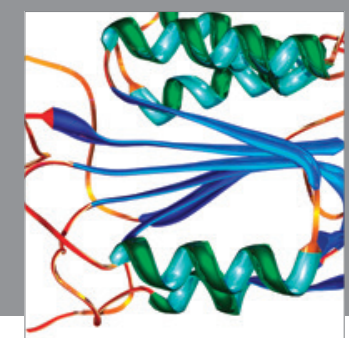

Disease Markers
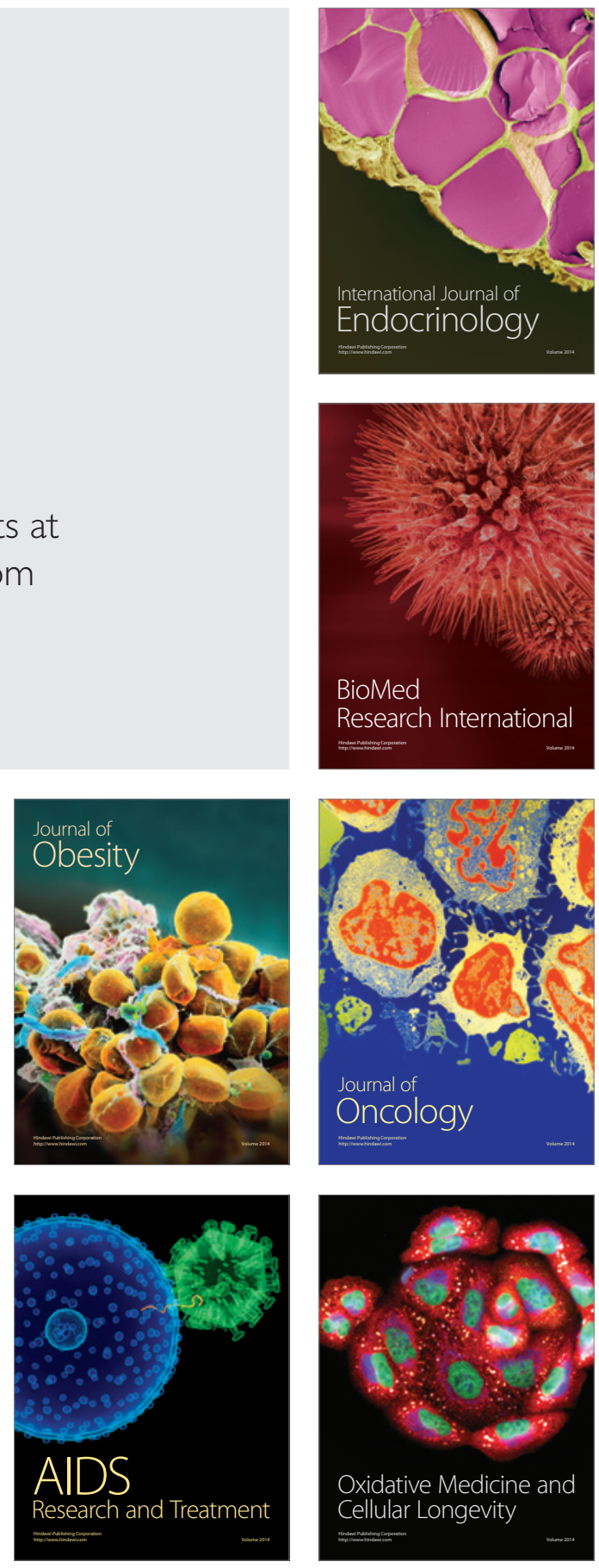